\title{
Selective bilateral internal iliac artery embolization for controlling refractory hematuria due to the metastatic squamous cell carcinoma of the urinary bladder: a case report
}

\author{
Gholamreza Mokhtari, ${ }^{1}$ Hossein Hemmati, ${ }^{1}$ Hamidreza Baghani Aval, ${ }^{2}$ Samaneh Esmaeili ${ }^{1}$ \\ ${ }^{1}$ Urology Research Center, Guilan University of Medical Sciences, Rasht; ${ }^{2}$ Sabzevar University of Medical Sciences, Sabzevar, \\ Iran
}

\begin{abstract}
Bladder squamous cell carcinoma (SCC) may lead to gross hematuria. However, the metastasis of head and neck cutaneous SCC to the urinary bladder has not been described in literature. Nowadays, noninvasive methods such as embolization, are considered as an appropriate choice for controlling life-threatening hematuria in patients with high operative risk. However, few reports exist on the effectiveness of this approach in managing the hematuria secondary to metastatic bladder SCC. Here we report a case of bladder SCC originating from the forehead cutaneous SCC. An 83-year-old man, a known case of forehead cutaneous SCC with distant metastasis, referred to our clinic with a chief complaint of hematuria. Pathology confirmed the diagnosis of metastatic urinary bladder SCC. Angiography and embolization were undertaken and resulted in complete alleviation of the symptoms. The recurrence of hematuria or embolization-related complications were not observed during 3-month follow-up. Selective embolization of the bilateral internal iliac artery is a safe and efficient procedure for controlling severe hematuria in patients with primary or metastatic bladder SCC.
\end{abstract}

\section{Introduction}

Bladder squamous cell carcinoma (SCC) is a rare type of bladder cancer, which frequently manifested

Correspondence: Hamidreza Baghani Aval, Sabzevar University of Medical Sciences, Sabzevar, Iran.

Tel./Fax: +98.1315525259.

E-mail: hamidreza_baghani@yahoo.com

Key words: Squamous cell carcinoma; bladder tumor; hematuria; embolization.

Acknowledgments: this study was supported by Urology Research Center, Guilan University of Medical Sciences.

Contributions: GM, study concept and design; HBA, SE, acquisition of data; GM, HH, HBA, SE, drafting of the manuscript; GM, HH, HBA, critical revision of the manuscript for important intellectual content; GM, HH, study supervision.

Conflict of interest: the authors have no conflict of interest.

Received for publication: 10 July 2016.

Accepted for publication: 30 August 2016.

This work is licensed under a Creative Commons Attribution NonCommercial 4.0 License (CC BY-NC 4.0).

(C) Copyright G. Mokhtari et al., 2017

Licensee PAGEPress, Italy

Italian Journal of Medicine 2017; 11:211-214

doi:10.4081/itjm.2016.761 with gross painless hematuria. ${ }^{1-4}$ Distant (lymphatic and hematogenous) metastases is unusual, ${ }^{1}$ and the metastasis of head and neck cutaneous SCC to the urinary bladder has rarely been previously reported in the literature, to the best of our knowledge. The surgery in older subjects with poor health conditions can increase the risk of morbidity and mortality, and also disrupt homeostasis, due to abnormal neovasculatures in the tumor. Therefore, because of unfavorable complications, surgery is not acceptable as an appropriate option in patients with higher stages (T4) or metastatic bladder tumors (BTs). ${ }^{2,3,5,6}$ Thus, the treatment of choice in most of patients with stage $4 \mathrm{BT}$ is palliative therapy. ${ }^{7}$ Embolization, which is a noninvasive procedure with safe features, has recently been used as an efficient method for alleviating of BT-related hematuria, ${ }^{3,5-7}$ but its efficacy in patients with bladder SCC is poorly been defined. Here, we present a known case of cutaneous SCC of head and neck who presented to our clinic with severe gross hematuria, and underwent selective artery embolization after the diagnosis of bladder SCC was assured.

\section{Case Report}

An 83-year-old man referred to urology clinic of our Hospital complaining of weakness, lethargy and gross total hematuria, with clot excretion as well as mild obstructive symptoms of lower urinary tract since 2 weeks ago. 5 years ago he presented to the dermatology clinic with a chief complaint of nonhealing 
ulcer. The diagnosis of cutaneous head and neck SCC was made based on clinical evolution and pathological biopsy. In addition, his left eye was extracted because of the presence of extensive invasion of the orbital cavity and pressure effect of metastatic mass. The patient then underwent a course of radiotherapy. After 3 years he presented to orthopedic clinic complaining of pathologic fracture and bone pain, after radiologic evaluation the involvement of head of femur due to SCC metastasis was shown and replacement of head of femur by prosthesis performed.

The patient did not have other medical problems and denied any previous or recent history of nephrolithiasis, urethral catheterization, or urologic surgery. He reported no allergies and took no medications such as cyclophosphamide. In addition, he was a farmer, and had no history of smoking and alcohol assumptions. On examination, genitourinary examination revealed mild benign prostate hyperplasia in digital rectal exam. The other examinations were unremarkable.
Laboratory tests showed decreased hemoglobin to $8.4 \mathrm{~g} / \mathrm{dL}$ and elevated serum creatinine $(\mathrm{Cr})$ of 2.7 $\mathrm{mg} / \mathrm{dL}$; as well as, total prostate-specific antigen of $0.9 \mathrm{ng} / \mathrm{dL}$. The additional laboratory tests including liver function tests were normal. Urinary analysis revealed gross hematuria (blood: 4+, red blood cells count: many), pyuria (white blood cells count: many). However, urinary culture was negative.

Ultrasonography (US) showed mild to moderate hydronephrosis of the left kidney and enlarged prostate gland (40-50 g), in addition to the relative increase in the left lateral bladder wall thickness with calcification.

There was no evidence of liver and lungs involvement in the chest X-ray. Non-contrast computer tomography of abdomen and pelvis revealed gallstone, free and abundant fluid in pelvic and abdominal cavities, hiatal hernia, left mild hydroureteronephrosis, fixation of left femoral intertrochanteric and neck fracture of the left femur. Left lateral bladder wall mass with calcification causing the ureteral obstruction is seen on the left

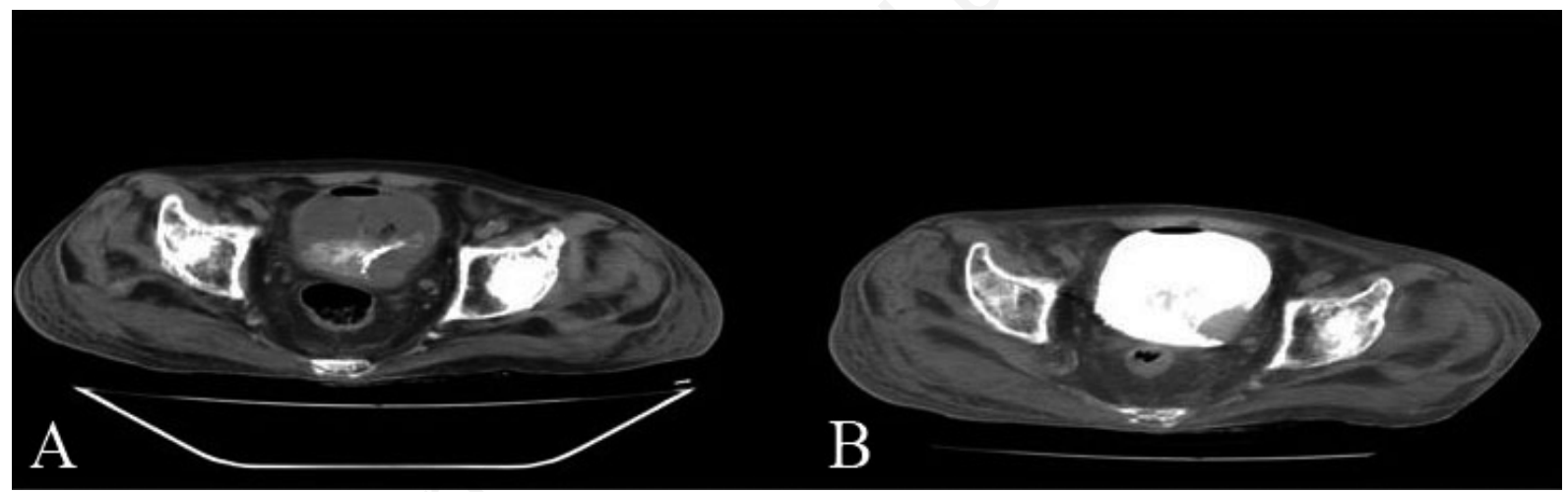

Figure 1. A) Non-contrast pelvic computed tomography (CT) scan; B) non-contrast CT cystography.

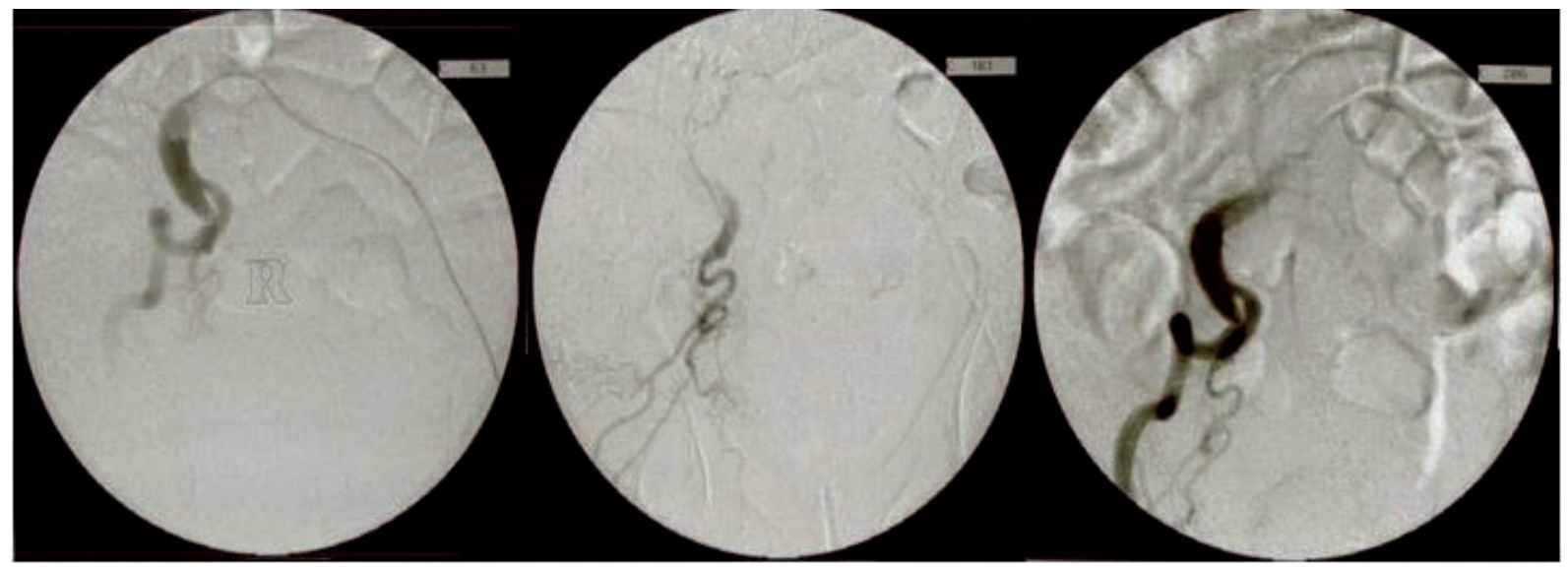

Figure 2. Images of angioembolization. 
(Figure 1). After sedation and local anesthesia, the patient underwent angiography of the aortoiliac and highly selective bilateral internal iliac artery embolizations of terminal branch in supine position using coil (Figure 2).

The cystoscopy was performed and revealed large calcified tumor in the left lateral wall of the bladder. Biopsy of the bladder mass and suspicious areas confirmed bladder SCC by showing malignant SCC cells.

After discharge, the patient was followed up for 1 week, 1 and 3 months later without complaining of procedure complications or recurrence of his previous symptoms. The patient's $\mathrm{Cr}$ decreased to 0.9 and his hemoglobin rose to 10.4 at a time of hospital discharge. Moreover, patients U/A were normal in every 3 times of follow-up. The controlled US in the third month did not show hydronephrosis and other abnormal findings.

\section{Discussion and Conclusions}

The occurrence of both head and neck and urinary bladder SCC, regardless of primary or metastatic form in that patient is an almost terrible disaster, which has rarely been described so far. In the only case report study to date, SCC of the urinary bladder occurred due to a metastasis from the oral cavity caused total painless hematuria with or without clot excretion. ${ }^{8}$ Additionally, few reports about performing embolization for alleviating the symptoms of urinary bladder SCC are available.

DNA microarrays and immunohistochemistry can differentiate the primary form of SCC from metastatic form. ${ }^{8}$ However, since the possibilities of molecular analysis of tumors in our center were not possible, besides, determining the type of tumor did not change our treatment plan, we did not investigate the underlying molecular mechanisms of tumor. So, although we cannot definitely claim that this tumor is caused by metastasis, the patient has several features that make primary bladder SCC less likely. Manifestations of primary bladder SCC usually occur earlier and affect tobacco users more commonly; however, the history of most of predisposing factors of primary bladder SCC including nephrolithiasis, diverticulum, long-term indwelling catheters, pelvic radiation, taking cyclophosphamide and schistosoma infection, ${ }^{7,8}$ were negative in our patient. In addition, historical sequences of our patient's symptoms are against the probability of primary bladder SCC.

Cystoscopy is recommended for all patients older than 60 years presenting with gross hematuria. In combination with cystoscopy, biopsy is a gold standard for diagnosing all types of bladder masses. ${ }^{7}$ The diagnosis of metastatic bladder SCC was established for our patients based on both cystoscopy and biopsy results.
Hematuria from bladder is a major clinical problem with difficult management strategies (challenges). ${ }^{1-3}$ The treatment of BT related hematuria depending on depth of tumor invasion, stage and general appearance ranges from medical to ablation therapy. ${ }^{2,7,9}$ However, surgery is not feasible in the later stages of BT and the main purpose of treatment in this stage is palliative therapy to alleviate symptoms by stopping life threatening hematuria. ${ }^{2,3,5}$ Transcatheter artery embolization can be a good substitute for highrisk surgical procedures, when we face with hematuria secondary to bladder malignancies, and failed to use routine procedures to stop bleeding. ${ }^{6}$

Recent studies have reported long-term successful benefits of embolization in control of severe hematuria due to the pelvic malignancies, BT and prostate cancer. ${ }^{3,5,6,10}$ It is recommended this procedure to be done before surgery in pelvic malignancies. ${ }^{2}$ Since our patient was elderly and suffered from end stage of bladder SCC, we undertake embolization to control his hematuria. Embolization of the internal iliac artery immediately stopped the bleeding. The patient follow-up showed beneficial effects of embolization in reducing symptoms of bladder SCC without major complication.

In conclusion, in dealing with the patients with positive history of head and neck SCC, the probability of its invasion of the genitourinary system should be considered by health care specialist. Thus, early detection of nodal invasion of tumor is of significant importance, and regular urological evaluations should be scheduled in patients with nodal metastasis. In addition, like other bladder masses, embolization was a feasible, safe and efficient procedure for controlling severe hematuria caused by primary or metastatic bladder SCC, and can be a good alternative to surgery in patients with high grade BTs.

\section{References}

1. Shokeir AA. Squamous cell carcinoma of the bladder: pathology, diagnosis and treatment. BJU Int 2004;93: 216-20.

2. Liguori G, Amodeo A, Mucelli FP, et al. Intractable haematuria: long-term results after selective embolization of the internal iliac arteries. BJU Int 2010;106:500-3.

3. Yu H, Guo Z, Xing W, et al. Selective internal iliac artery oxaliplatin infusion: another facultative treatment to unremitting hematuria in stage T4 bladder carcinoma. Clin Oncol Cancer Res 2010;7:55-9.

4. Kaufman DS, Shipley WU, Feldman AS. Bladder cancer. Lancet 2009;374:239-49.

5. Delgal A, Cercueil JP, Koutlidis N, et al. Outcome of transcatheter arterial embolization for bladder and prostate hemorrhage. J Urol 2010;183:1947-53.

6. El-Assmy A, Mohsen T. Internal iliac artery embolization for the control of severe bladder hemorrhage secondary to carcinoma: long-term follow-up. Sci World J 2007;7:1567-74. 
7. Wong JT, Wasserman NF, Padurean AM. Bladder squamous cell carcinoma. RadioGraph 2004;24: 855-60.

8. Goel T, Reddy S, Thomas J, Garg S. Squamous cell carcinoma of the oral cavity with metastasis to the urinary bladder. Indian J Urol 2006;22:372-3.

9. Stenzl A, Cowan NC, De Santis M, et al. Treatment of muscle-invasive and metastatic bladder cancer: update of the EAU Guidelines. Eur Urol 2011;59:1009-18.

10. Nabi G, Sheikh N, Greene D, Marsh R. Therapeutic transcatheter arterial embolization in the management of intractable haemorrhage from pelvic urological malignancies: preliminary experience and long-term follow-up. BJU Int 2003;92:245-7. 